\title{
Development of a device to increase the protection of vulnerable road users in the case of impact against heavy vehicles
}

\author{
M. Avalle, G. Belingardi \& A. Giorda \\ Department of Mechanics, Politecnico di Torino, Italy
}

\begin{abstract}
The protection of vulnerable road users in urban traffic is nowadays a major issue in vehicle construction. Numerical techniques to perform virtual tests are of great help in dealing with these vehicle design problems.

The objective of this research is the selection of an optimal configuration for a pedestrian protection system based on the adaptive surfaces in Heavy Vehicles (HV). These adaptive surfaces can be an add-on solution for the front grille and bumper, and are moved by means of actuators in pre-impact situations.

A numerical multibody model has been developed with MADYMO code to simulate a $30 \mathrm{~km} / \mathrm{h}$ truck-pedestrian impact. This model has been used to set-up a full factorial plan with three variables in order to reduce the possible biological damage to the pedestrian. The damage risk is evaluated by means of the Head Injury Criterion (HIC). The optimal solution with respect to the three variables has been determined by means of a regression model.
\end{abstract}

Keywords: passive safety, optimisation, adaptive surfaces, pedestrian impact, heavy vehicles.

\section{Introduction}

Pedestrian impact study is currently a relevant topic in vehicle safety design $[1,2]$. The goal of approval rules such as the $2003 / 102 / \mathrm{CE}$ directive that is compulsory for all new vehicles starting from 2010, and rating rules such as EuroNCAP tests [3] is to improve the passive safety performance of the vehicles, with particular attention to the VRUs (Vulnerable Road Users) such as pedestrians.

Meeting these safety requirements means reducing the aggressivity of the external vehicle structure against pedestrians in order to limit as much as 
possible biomechanical injuries that can come from vehicle-structure interaction [3-5].

Limiting injuries to pedestrians is a very difficult task due to the considerable vehicle mass and front stiffness involved in accidents between pedestrians and trucks [6-9]. Geometry also has great importance due to the typical European truck frontal conformation, easily represented by a vertical wall. In this case all the pedestrian body sections are almost contemporarily interested, while in a pedestrian to car accident the lower extremities are first interested and then the remaining body sections. The accident event is particularly unfavourable because in this way the head is involved early in the impact, with the maximum collision energy and the greatest risks for the pedestrian survival.

The goal of this research work consists of searching the best configuration of the truck front components, to obtain a lower aggressivity using adaptive surfaces. Adaptive surfaces can be the covers of the frontal panel and bumper and can be positioned by means of actuators (figure 1). The surfaces can be actuated in urban viability movement conditions in order to protect pedestrians in accident situations using their most favourable geometric configuration. This is one of the solutions studied in the subproject SP2 (Heavy Vehicles) of the European Project APROSYS, dedicated to the protection improvement of VRUs (pedestrians and cyclists).

\section{Adaptive surfaces}

Adaptive surfaces are composed of a front panel module and a bumper module that are movable by means of actuators as shown in figure 1. The front panel module is constrained to the heavy vehicle structure by means of hinges positioned at the upper side of the panel. The actuator pushes forward the lower side of the panel, thus the surface rotates around the upper horizontal axis (hinge axis). The bumper module surface is constrained to a second actuator at its centre of gravity; this second actuator provides the forward movement and the rotation movement around a horizontal axis parallel to the front panel horizontal axis and positioned at the constraint.
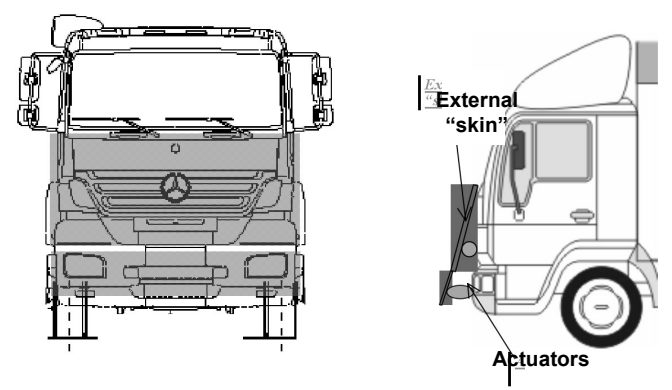

Figure 1: $\quad$ Adaptive surfaces working scheme. 
Adaptive surfaces provide an additional impact gap (up to $100 \mathrm{~mm}$ ) at the pelvis and thorax regions. They also introduce a modification to the pedestrian kinematic due to the impact: this fact reduces run-over risk and the severity of the secondary impact with the road. The adaptive surface modules have to be considered useful for speeds up to $60 \mathrm{~km} / \mathrm{h}$.

\section{Impact model}

An impact accident between a heavy vehicle and a vulnerable road user has been reconstructed through a multibody numerical model built in the MADYMO environment. The heavy vehicle has been modelled with the frontal skin of an IVECO STRALIS truck. This model consists of facet elements that are rigidly connected to the support body (light grey part shown in figure 2). Two panels, modelled by perfectly rigid ellipsoids that represent the adaptive surfaces, have been added to the vehicle as shown in figure 2 (dark grey components). The vulnerable road user is modelled by means of a standard virtual human model in standing configuration.
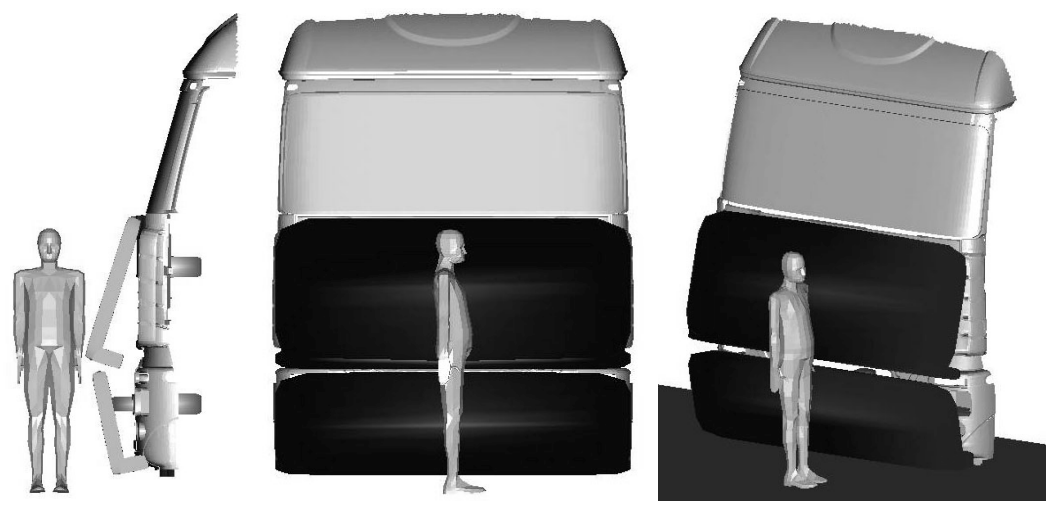

Figure 2: Adaptive surfaces and pedestrian position.

The particular case of impact analysed in the performed simulation reproduces the pedestrian impacted by the truck: the pedestrian is positioned in the middle of the width of the truck front. Figure 2 shows the pedestrian's initial position before the impact: the sagittal pedestrian plane is perpendicular to the vehicle driving direction. The initial vehicle speed used in the simulation is 30 $\mathrm{km} / \mathrm{h}$.

\section{Methodology}

A complete factorial plan has been developed in order to study the influence of the front panel and bumper adaptive surfaces position. This DOE plan involves three independent variables that control the position and the rotation of the adaptive surfaces with respect to the original frontal profile. The analysed 
response is the HIC biomechanical parameter. A regression analysis has been set up to search for the optimal configuration [10]. The three variables taken into consideration are shown in figure 3 , the variation constraints are:

- Front panel rotation: rotation angle between 0 and $0.3 \mathrm{rad}$.

- Bumper displacement: displacement range between 0 and $0.2 \mathrm{~m}$.

- Bumper rotation: rotation angle between -0.3 and $0.3 \mathrm{rad}$.
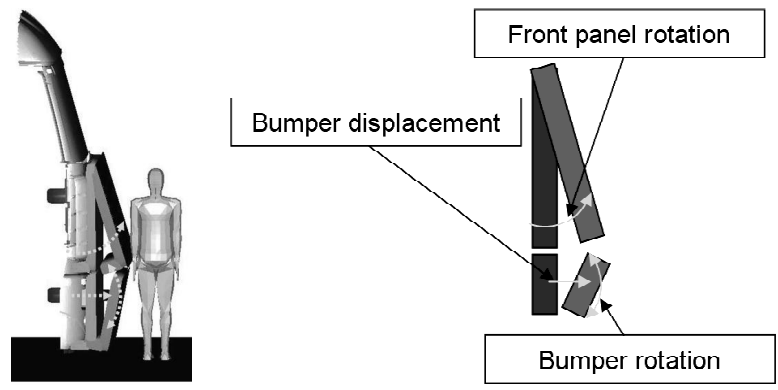

Figure 3: Adaptive surfaces in the reference configuration (light grey) and in a generic actuated configuration (dark grey).

The complete factorial plan of simulations consists of 140 simulations as described in the following tables:

- 4 levels for front panel rotation:

\begin{tabular}{|l|l|l|l|l|}
\hline Rotation (rad) & 0 & 0.1 & 0.2 & 0.3 \\
\hline
\end{tabular}

- 5 levels for the bumper panel displacement:

\begin{tabular}{|l|l|l|l|l|l|}
\hline Displacement $(\mathrm{m})$ & 0 & 0.05 & 0.1 & 0.15 & 0.2 \\
\hline
\end{tabular}

- 7 levels for the bumper panel rotation:

\begin{tabular}{|l|l|l|l|l|l|l|l|}
\hline Rotation (rad) & -0.3 & -0.2 & -0.1 & 0 & 0.1 & 0.2 & 0.3 \\
\hline
\end{tabular}

A non-linear regression analysis allowed determination of the 68 coefficients of a polynomial expression used to describe the four dimension response surfaces with respect to the design variables.

\section{Results}

The correlation between the values of the HIC parameter estimated by means of the regression analysis and the values calculated by means of the numerical model is shown in figure 4 . The analysis of the diagram of figure 4 provides evidence of little dispersion of the results: the points are in general significantly close to the $45^{\circ}$ straight line that represents the perfect correlation between estimated and predicted values.

Figure 5 shows the influence of each variable on the response (ANOVA). It is evident that the front panel rotation plays a fundamental role and the response behaviour is very sensitive to this variable. On the contrary, the variables referring to bumper position (displacement and rotation) have an influence of less than 10\%: so it is possible to disregard these two latter variables (or at least the bumper rotation) with little increase in error. 


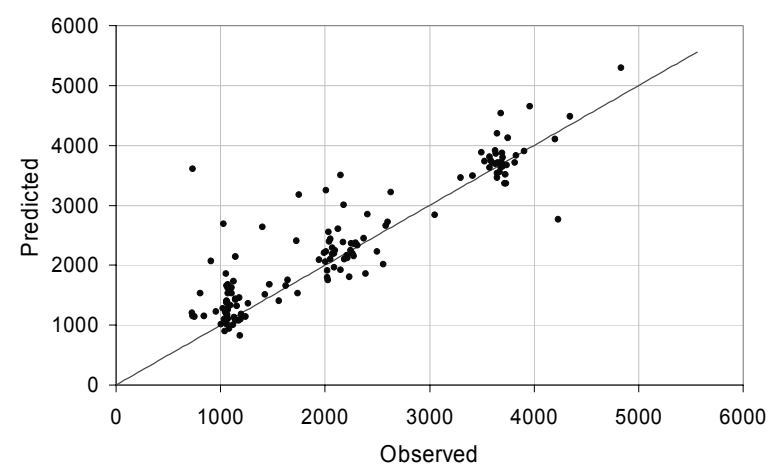

Figure 4: $\quad$ Comparison of predicted and observed results.

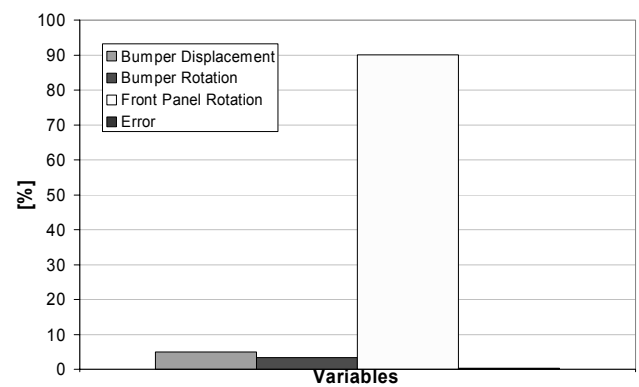

Figure 5: $\quad$ Relevance of the three design variables.

A four dimensional space would be necessary in order to represent the results of the complete factorial plan as there are three variables and one response. For clarity of result representation it is convenient to show the response value as a function of two variables in turn using the third variable as a parameter. In particular it is convenient to plot the response surface for each value of the bumper rotation, thus obtaining seven three dimensional surfaces which represent the slices of the whole response volume. Figure 6 shows, for example, the three dimensional response surface obtained for the 0 rad bumper panel rotation.

There is a similar trend for the surfaces obtained for each considered value of the bumper rotation: the minimum values of the HIC parameter are reached in correspondence of large bumper panel displacements combined with large front panel rotations. HIC minima are obtained for the front panel rotation close to 0.3 rad and the bumper panel displacement close to $0.2 \mathrm{~m}$, as shown in table 1 . The bumper rotation gives slightly different values in each minimum configuration (highlighted in table 1), this confirms the little influence of this design variable.

The configuration used in simulation 128 gives the absolute minimum configuration: it corresponds to $0.2 \mathrm{~m}$ bumper panel displacement, 0 rad bumper panel rotation and $0.3 \mathrm{rad}$ front panel rotation. 


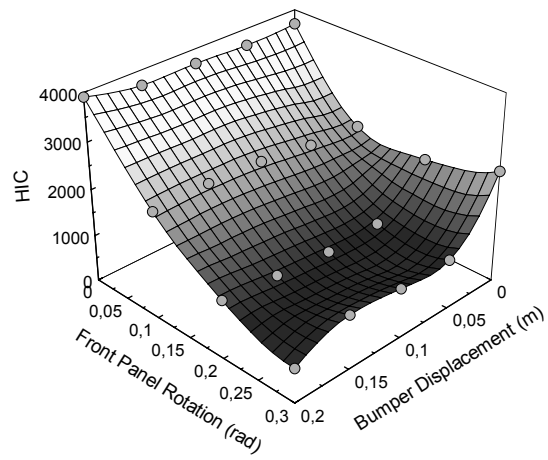

Figure 6: Response surface for the $0 \mathrm{rad}$ bumper rotation.

Table 1: $\quad$ The best obtained results.

\begin{tabular}{|c|c|c|c|c|}
\hline Test & $\begin{array}{c}\text { Bumper } \\
\text { displacement }(\mathrm{m})\end{array}$ & $\begin{array}{c}\text { Bumper } \\
\text { rotation (rad) }\end{array}$ & $\begin{array}{c}\text { Frontal panel } \\
\text { rotation (rad) }\end{array}$ & HIC \\
\hline 67 & 0.10 & -0.1 & 0.2 & 1004.5 \\
\hline 92 & 0.15 & -0.2 & 0.3 & 803.8 \\
\hline 96 & 0.15 & -0.1 & 0.3 & 839.7 \\
\hline 111 & 0.15 & 0.3 & 0.2 & 958.9 \\
\hline 116 & 0.20 & -0.3 & 0.3 & 733.3 \\
\hline 119 & 0.20 & -0.2 & 0.2 & 907.9 \\
\hline 124 & 0.20 & -0.1 & 0.3 & 751.9 \\
\hline 128 & 0.20 & 0.0 & 0.3 & 729.5 \\
\hline 132 & 0.20 & 0.1 & 0.3 & 734.6 \\
\hline
\end{tabular}

\subsection{Biomechanical results of the simulations involving the human model}

Some biomechanical parameters have been taken into consideration in order to analyse the biomechanical behaviour of the pedestrian during the accident: the HIC parameter, the maximum head acceleration, the maximum resultant hip force, the maximum resultant knee force and the maximum resultant sacrum force. Two different configurations are compared for each biomechanical parameter: a reference configuration corresponding to the simulation without displacements and rotations and the optimal (absolute minimum) configuration. Only the results that refer to HIC, maximum head acceleration and sacrum maximum resultant force are reported here because they show a behaviour much more critical than the other parameters.

\subsubsection{Head acceleration}

Resultant head acceleration in the reference configuration is very high in the first impact with the heavy vehicle but also in the second impact with the road plane. The maximum acceleration level falls down for the optimal configuration as shown in figure 7 where the most severe acceleration peak, at the first impact, is put in evidence on the right. 
The calculated HIC value is considerably different between the two configurations: a bewildering value of 3695 has been obtained in the reference configuration while a more favourable value of 729 has been obtained in the optimal configuration.

\subsubsection{Sacrum resultant force}

Figure 8 shows the resultant pelvis-sacrum force trend. In this case it can be noted that the maximum resultant force is greater in the optimal configuration than in the reference. Therefore, this choice of adaptive surfaces position does not succeed in reducing damage in the pelvis-sacrum region.

It should be noted that this parameter was not considered in the optimum configuration search.
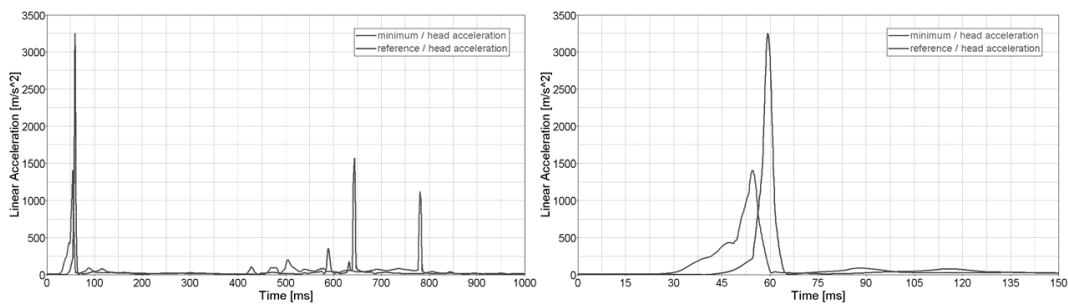

Figure 7: Head acceleration: right, zoom of the first impact.
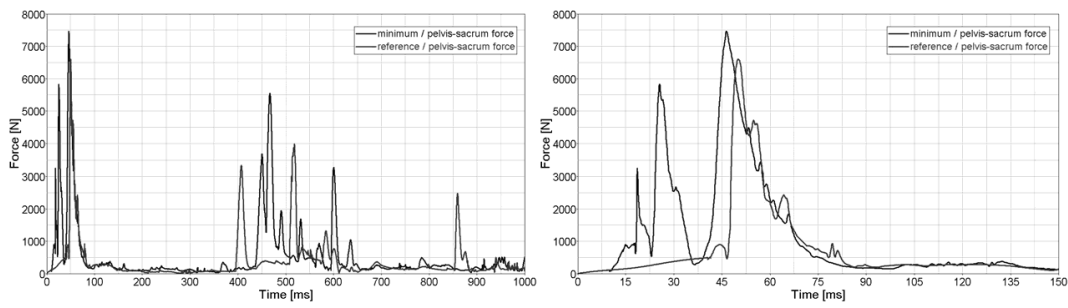

Figure 8: Pelvis-sacrum region force: right, zoom of the first impact.

\subsubsection{Reference and absolute minimum configuration comparison}

Figure 9 shows the comparison between the kinematic of the reference (light grey) and the optimal configuration (dark grey): the head of the virtual human model in the optimal configuration is subjected to a lower impact force than in the reference configuration.

\section{Further development of the design}

A development of the model has been obtained by the introduction of the deformability of the impact surface. The basic idea consists of covering the surfaces with a layer of structural foam that is able to absorb a relevant part of the total impact energy [11]. For this purpose two deformable layers, built by means of FE and corresponding to the rigid panels, have been introduced into the base multibody model (figure 10). 
Two different material models derived from experimental tests obtained by Avalle et al. [12] have been taken into consideration. The calculated HIC values are reduced even more with adaptive deformable surfaces due to the absorption of part of the head impact energy by the deformable layer. The calculated HIC value using an EPP45 polypropylene structural foam is reduced to 673 while the value obtained using a PUR100 polyurethane structural foam is 683. In both cases, the values are lower than the value of 729 calculated for the same minimum configuration using rigid surfaces. The maximum head acceleration level is reduced using the deformable surfaces in accordance to what happens to HIC values as shown in figures 11 and 12 .

The use of deformable surfaces allows large improvements to be obtained especially in the pelvis-sacrum region where, for the case of the rigid surface model, a resultant maximum force value close to $7.5 \mathrm{kN}$ was calculated.

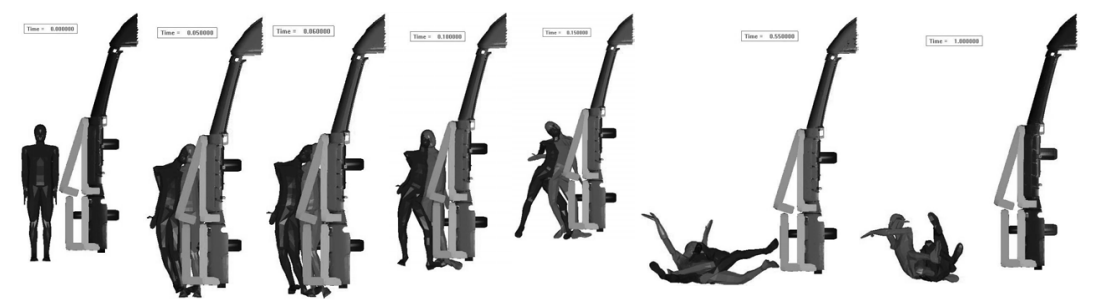

Figure 9: Impact sequence: reference configuration (light grey) and minimum configuration (dark grey).
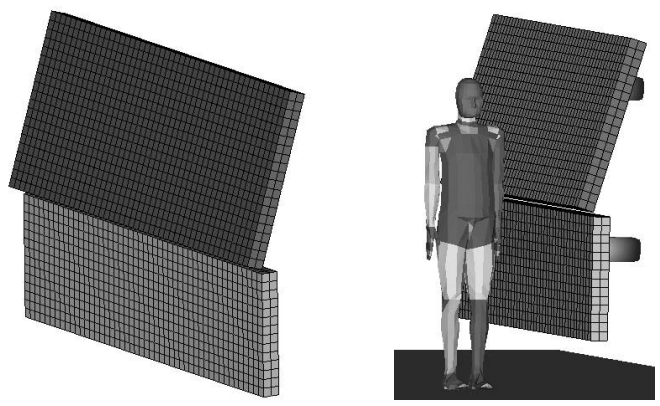

Figure 10: Adaptive deformable surfaces.
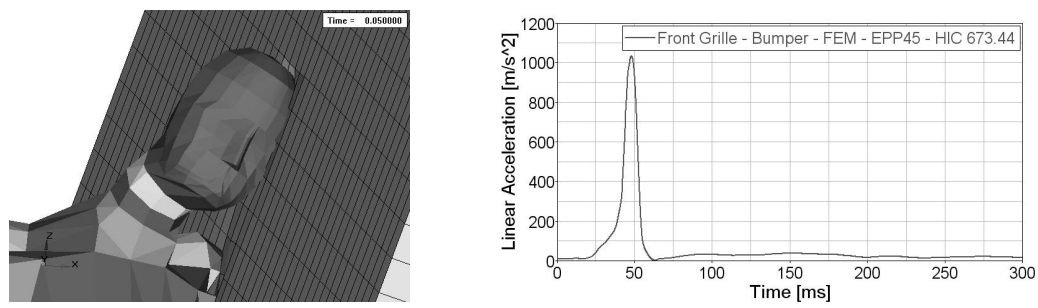

Figure 11: Maximum head acceleration (EPP45). 
Figure 13 shows the improvement of protection obtained using foams: the resultant force reaches a maximum value close to $2.25 \mathrm{kN}$ with the PUR100 foam and close to $1.5 \mathrm{kN}$ with the EPP45 foam.

The impact sequence reported in figure 14 shows the deformable surface deformation due to pedestrian impact. The repulsion action on the pedestrian is reduced by the energy absorbed in the foam deformation.
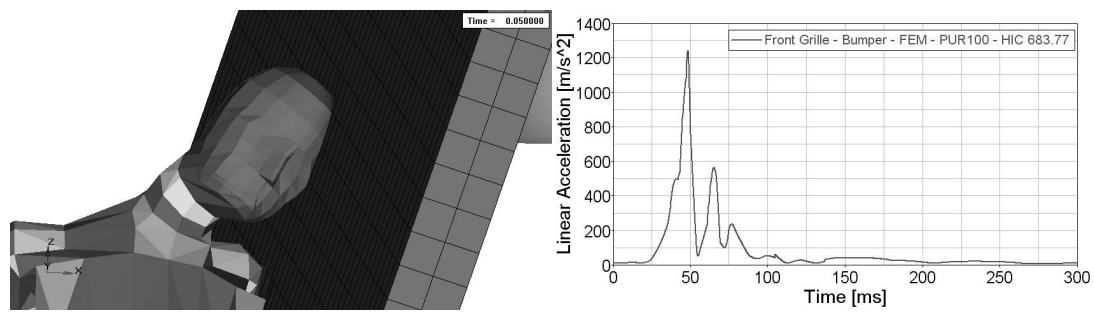

Figure 12: Maximum head acceleration (PUR100).
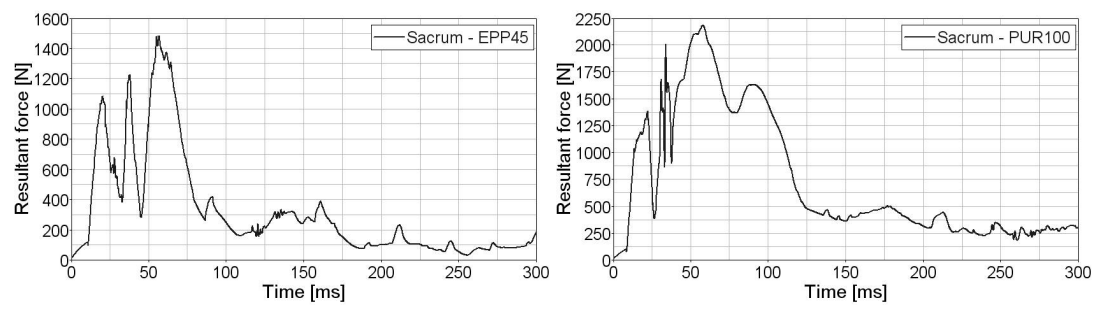

Figure 13: Maximum resultant force of the Pelvis-sacrum region.

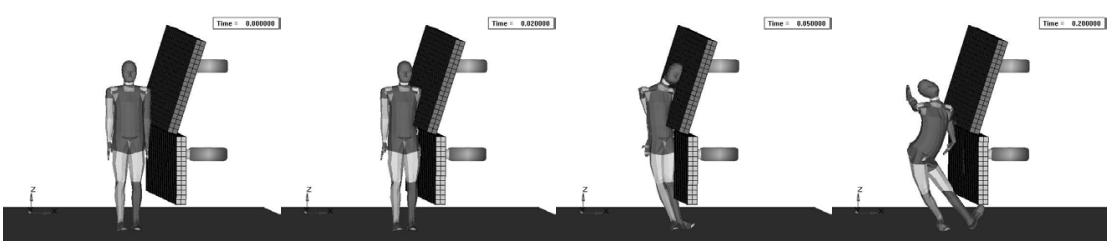

Figure 14: Impact sequence.

\section{Conclusions}

The event of the pedestrian to heavy truck impact has been examined in order to design solutions to reduce the consequent injury level for the pedestrian. A front panel with adaptive surfaces has been designed. Three design variables have been considered, the assumed objective function to be optimised was the HIC parameter. The optimal solution has been searched through a complete factorial plan, explored in a virtual environment by means of a numerical multibody model built with MADYMO code.

The more effective design variable is the rotation of the front panel; the bumper panel displacement has only limited influence on the results, while the third variable, the bumper panel rotation, has practically no affect on the results. 
The optimum protection level of the pedestrian has been reached by means of a configuration with a frontal panel rotation equal to $0.3 \mathrm{rad}$ and a bumper panel displacement of $0.2 \mathrm{~m}$ : the calculated HIC value is 729 . A very large improvement has been obtained with respect to the reference solution where the HIC value resulted to be something less than 3700 .

However, the use of rigid surfaces causes severe injury in the pelvis-sacrum region. The introduction of deformable surfaces also allows a large reduction of the maximum values for this biomechanical parameter together with a further reduction of the HIC and the maximum head acceleration values.

The use of the EPP45 structural foam leads to better results, i.e. lower levels of each of the considered biomechanical parameters, than the use of the PUR100.

\section{References}

[1] Lefler, D.E., Gabler, H.C., The emerging threat of light truck impacts with pedestrians. Proc. of the $17^{\text {th }}$ ESV Conf., paper No. 212, 2001.

[2] Smith, T., Broughton, J., Knight, I., Passenger, goods and agricultural vehicle safety - effectiveness of existing measures and ranking of future priorities in the UK. Proc. of the 20 th ESV Conf., paper 07-0452, 2007.

[3] EuroNCAP. Pedestrian Testing Protocol, Version 4.1, 2004.

[4] EEVC Working Group 17: Improved test methods to evaluate pedestrian protection afforded by passenger cars, http://www.eevc.org, 2002.

[5] Janssen, E.G., EEVC test methods to evaluate pedestrian protection afforded by passengers cars. Proc. of the $15^{\text {th }}$ ESV Conf., paper 96-S7-W$17,1996$.

[6] Pipkorn, B., Fredriksson, R., Olsson, J., Bumper bag for SUV to passenger vehicle compatibility and pedestrian protection. Proc. of the $20^{\text {th }}$ ESV Conf., paper 07-0056, 2007.

[7] Lee, K.B., Jung, H.J., Bae, H.I., The study on developing active hood lift system for decreasing pedestrian head injury. Proc. of the $20^{\text {th }}$ ESV Conf., paper 07-0198, 2007.

[8] Nagatomi, K., Hanayama, K., Ishizaki, T., Sasaki, S., Matsuda, K., Development and full-scale dummy tests of a pop-up hood system for pedestrian protection. Proc. of the $19^{\text {th }}$ ESV Conf., paper 05-0113, 2005.

[9] Enomoto, H., Akiyama, K., Development of safety concept trucks; ASV concept L and ASV concept C. Proc. of the $19^{\text {th }}$ ESV Conf., paper 05-0295, 2005.

[10] Belingardi, G., Chiandussi, G., Giorda, A., Multi-point optimisation methodologies applied to design problems oriented to car driver protection. Proc. of the $9^{\text {th }}$ Florence ATA Conf., 2005.

[11] Belingardi, G., Duella, R., Caminiti, A., Optimal choice of the foam design parameters in order to meet the HIC index limit of the FMVSS201 standard. Proc. of the 2002 IBEC Conf., paper 2002-01-2095, 2002.

[12] Avalle, M., Belingardi, G., \& Ibba, A., Mechanical models of cellular solids: parameters identification from experimental tests. International Journal of Impact Engineering, 34, pp. 3-27, 2007. 